\title{
Densities, infrared band strengths, and optical constants of solid methanol
}

\author{
Ramón Luna ${ }^{1}$, Germán Molpeceres $^{2}$, Juan Ortigoso ${ }^{2}$, Miguel Angel Satorre ${ }^{1}$, Manuel Domingo ${ }^{1}$, and Belén Maté ${ }^{2}$ \\ ${ }^{1}$ Centro de Tecnologías Físicas, Universitat Politècnica de València, Plaza Ferrándiz-Carbonell, 03801 Alcoy, Spain \\ 2 Instituto de Estructura de la Materia, IEM-CSIC, Serrano 123, 28006 Madrid, Spain \\ e-mail: belen.mate@csic.es
}

Received 21 May 2018 / Accepted 29 June 2018

\begin{abstract}
Contact. The increasing capabilities of space missions like the James Webb Space Telescope or ground-based observatories like the European Extremely Large Telescope demand high quality laboratory data of species in astrophysical conditions for the interpretation of their findings.

Aims. We provide new physical and spectroscopic data of solid methanol that will help to identify this species in astronomical environments.

Methods. Ices were grown by vapour deposition in high vacuum chambers. Densities were measured via a cryogenic quartz crystal microbalance and laser interferometry. Absorbance infrared spectra of methanol ices of different thickness were recorded to obtain optical constants using an iterative minimization procedure. Infrared band strengths were determined from infrared spectra and ice densities.

Results. Solid methanol densities measured at eight temperatures vary between $0.64 \mathrm{~g} \mathrm{~cm}^{-3}$ at $20 \mathrm{~K}$ and $0.84 \mathrm{~g} \mathrm{~cm}^{-3}$ at $130 \mathrm{~K}$. The visible refractive index at $633 \mathrm{~nm}$ grows from 1.26 to 1.35 in that temperature range. New infrared optical constants and band strengths are given from 650 to $5000 \mathrm{~cm}^{-1}(15.4-2.0 \mu \mathrm{m})$ at the same eight temperatures. The study was made on ices directly grown at the indicated temperatures, and amorphous and crystalline phases have been recognized. Our optical constants differ from those previously reported in the literature for an ice grown at $10 \mathrm{~K}$ and subsequently warmed. The disagreement is due to different ice morphologies. The new infrared band strengths agree with previous literature data when the correct densities are considered.
\end{abstract}

Key words. solid state: volatile - methods: laboratory: molecular - techniques: spectroscopic - ISM: abundances - infrared: ISM infrared: planetary systems

\section{Introduction}

Ice mantles on dust grains in dense clouds in the interstellar medium (ISM) mainly consist of $\mathrm{H}_{2} \mathrm{O}, \mathrm{CO}$, and $\mathrm{CO}_{2}$. Although it varies from source to source, methanol is in many of them the fourth most abundant species, with an abundance of $6 \%$ with respect to water. Slightly lower amounts of $\mathrm{NH}_{3}$ and $\mathrm{CH}_{4}$ are also present (Boogert et al. 2015). Methanol is formed on the surface of the grains by hydrogenation of $\mathrm{CO}$ to form $\mathrm{H}_{2} \mathrm{CO}$ and then $\mathrm{CH}_{3} \mathrm{OH}$ (Watanabe \& Kouchi 2002; Ioppolo et al. 2011). The distribution of these species in the ice mantle is not homogenous and probably two different layers exist, an inner water-rich layer containing most of the $\mathrm{CO}_{2}$ and $\mathrm{NH}_{3}$, covered by an external water-poor layer formed of $\mathrm{CO}$, the remaining $\mathrm{CO}_{2}$, and most of the $\mathrm{CH}_{3} \mathrm{OH}$ (Öberg 2016). In particular, the possibility that pure $\mathrm{CH}_{3} \mathrm{OH}$ islands are segregated in the water-poor ice layer has been considered by some authors (Bottinelli et al. 2010).

Solid methanol has also been detected on the surface of minor bodies in the solar system, like some trans-Neptunian objects (TNOs) and Centaurus (Merlin et al. 2012). In Enceladus, one of the moons of Saturn, the presence of methanol ice was reported by Hodyss et al. (2009) based on an absorption feature appearing at $3.53 \mu \mathrm{m}\left(233 \mathrm{~cm}^{-1}\right)$ in an infrared spectrum of its surface registered by Cassini. Recently, the detection of a methanol cloud in Enceladus has been reported (Drabek-Maunder et al. 2017), which strengthens the possibility of having this species in solid phase on the surface.
Information about the composition and structure of astrophysical ices, and in particular the presence of solid methanol, is obtained by comparing astrophysical observations in the infrared to laboratory data (Dartois et al. 1999; Pontoppidan et al. 2003; Boogert et al. 2008; Bottinelli et al. 2010). The main infrared absorption features used for solid $\mathrm{CH}_{3} \mathrm{OH}$ identification in interstellar ices are the $3.53 \mu \mathrm{m}\left(2833 \mathrm{~cm}^{-1}\right)$ and $9.74 \mu \mathrm{m}\left(1027 \mathrm{~cm}^{-1}\right)$ bands. They correspond to the $\mathrm{CH}_{3}$ symmetric stretching mode and the $\mathrm{CO}$ stretching mode, respectively. The laboratory data most widely used for comparison to observations are those provided by d'Hendecourt \& Allamandola (1986), Hudgins et al. (1993), and Kerkhof et al. (1999). The first reference gives IR spectra and band strengths of methanol ice at $10 \mathrm{~K}$ in the $2.5-20 \mu \mathrm{m}$ range. The second reference presents a thorough infrared study providing a compendium of band strengths and optical constants in the 2.5-200 $\mu \mathrm{m}$ interval for ices of astrophysical relevance. For pure methanol, optical constants are given for an ice grown at $10 \mathrm{~K}$ and subsequently warmed to $50 \mathrm{~K}, 75 \mathrm{~K}$, $100 \mathrm{~K}$, and $120 \mathrm{~K}$. Band strengths at $10 \mathrm{~K}$ are also given. As far as we know, these are the only optical constants available in the literature for solid methanol. The third reference reports band strength variations of methanol absorptions in the mid-IR region with dilution in $\mathrm{H}_{2} \mathrm{O}$ ice, or in $\mathrm{H}_{2} \mathrm{O}$ and $\mathrm{CO}_{2}$ ice mixtures. Additional laboratory works provide infrared band strengths of this species at 10 K. Specifically, the data in Palumbo et al. (1999) correspond to the mid-IR (MIR), and the data in Sandford \& Allamandola (1993) and Gerakines et al. (2005) to the near-IR (NIR). Finally, 
a recent paper by Bouilloud et al. (2015) presents a compilation and new measurements of MIR band strengths of methanol ice at low temperatures. Since methanol ice densities were unknown, the uncertainty on band-strength determination is very large. In particular, a discrepancy among the literature results up to $40 \%$ for the strength of the $9.74 \mu \mathrm{m}$ band has been pointed out.

Our goal is to provide new data for solid methanol by obtaining, for the first time, actual density values for background deposited ices and by completing the available spectroscopic information on this species in the NIR and MIR spectral ranges. Thus, we have measured densities and the visible refractive index at $633 \mathrm{~nm}$ for methanol ices grown at $20 \mathrm{~K}, 40 \mathrm{~K}, 60 \mathrm{~K}, 80 \mathrm{~K}$, $100 \mathrm{~K}, 110 \mathrm{~K}, 120 \mathrm{~K}$, and $130 \mathrm{~K}$. We have evaluated IR band strengths and optical constants of solid methanol at those temperatures in the MIR and NIR spectral ranges from $650 \mathrm{~cm}^{-1}$ to $5000 \mathrm{~cm}^{-1}(15.4 \mu \mathrm{m}$ to $2.0 \mu \mathrm{m})$.

This paper is organized as follows: the experimental set-up and the procedures to determine densities, visible refractive index, infrared optical constants and band strengths are explained in Sect. 2; results are presented in Sect. 3; Sect. 4 is devoted to a discussion of the results in comparison with previous studies; and finally, our conclusions are summarized in Sect. 5.

\section{Experiments and data analysis}

Our experiments were performed using two different experimental set-ups, one at the UPV-Alcoy and the other at IEM-CSIC-Madrid. Both have been described in detail in previous publications (Satorre et al. 2017; Molpeceres et al. 2016; Zanchet et al. 2013).

The set-up at Alcoy allows us to determine densities and visible refractive indices. Densities are obtained by dividing the mass deposited in a certain area $\left(\mathrm{g} \mathrm{cm}^{-2}\right)$ by the thickness of the ice formed. The mass is determined by a quartz crystal microbalance (QCMB, $5 \mathrm{MHz}$ AT-cut Q-Sense) that follows the Sauerbrey equation (Sauerbrey 1959),

$\Delta F=-S \Delta m$,

where $\Delta F$ is the frequency variation (Hz) (right axis in Fig. 1), $S$ is a constant $\left(\mathrm{g} \mathrm{cm}^{-2} \mathrm{~Hz}^{-1}\right)$, and $\Delta m$ is the mass deposited $(\mathrm{g})$. Thickness was calculated by laser interferometry from the following expression giving the increase in thickness corresponding to one maximum of interference,

$$
\Delta x=\frac{\lambda_{0}}{2 n_{0} \sqrt{1-\left(\frac{\sin \alpha}{n_{0}}\right)^{2}}},
$$

where $\lambda_{0}$ is the wavelength of the He-Ne laser $(633 \mathrm{~nm}), n_{0}$ is the refractive index of the ice film at the laser wavelength, and $\alpha$ the incidence angle of the laser from the surface normal. For most ices formed at low temperatures $n_{0}$ is unknown. Two interference patterns were obtained at two different angles of incidence ( $34^{\circ}$ and $64^{\circ}$, left axis Fig. 1), giving us a second equation to solve two unknowns. The refractive index can be obtained directly from

$n^{2}=\frac{\sin ^{2} \beta-\gamma^{2} \sin ^{2} \alpha}{1-\gamma^{2}}$,

where $\gamma^{2}=\frac{T_{\alpha}}{T_{\beta}}$ and $T_{\alpha}$ and $T_{\beta}$ are the periods of the corresponding angles of incidence measured in seconds.

Films were formed at constant temperature allocating the QCMB at the end of the second stage of a Leybold closed-cycle

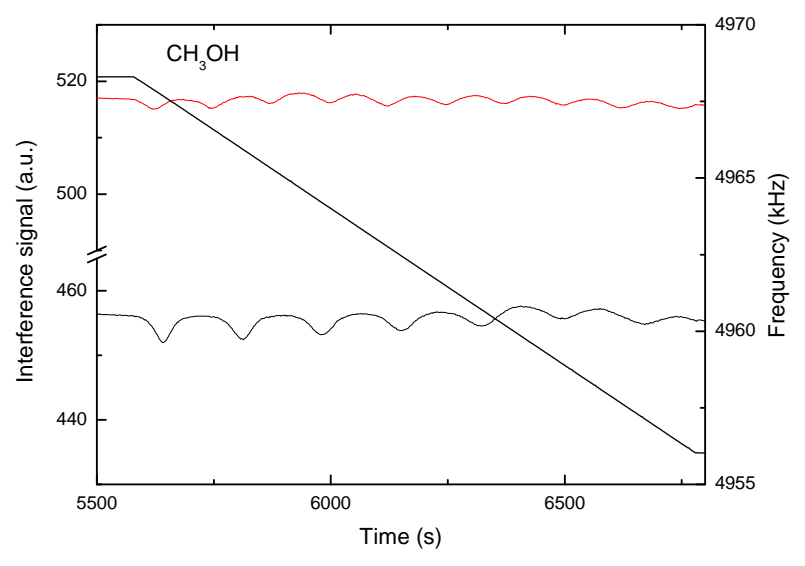

Fig. 1. Laser signals at two angles of incidence (left axis) and straight variation of the frequency of a QCMB (right axis) during methanol deposition at $80 \mathrm{~K}$ at constant rate.

He cryostat, where the temperature could be controlled from $12 \mathrm{~K}$ to room temperature by an ITC Oxford temperature controller. In these experiments, the temperature was fixed within $1 \mathrm{~K}$. Cold parts were surrounded by a radiation shield inside a high vacuum chamber to prevent contaminants in the QCMB. The pressure in the chamber increases from $5 \times 10^{-8} \mathrm{mbar}$ before accretion to a constant value of $10^{-5} \mathrm{mbar}$ during the deposition. Methanol molecules pass from a prechamber to the deposition chamber through a needle valve. Methanol pressure in the prechamber is maintained constant at 18.7 mbar thanks to both a liquid methanol reservoir at constant temperature and a controlled flux, through another needle valve, from the reservoir to the prechamber. These conditions lead to a deposition rate of $\approx 1 \mathrm{~nm} \mathrm{~s}^{-1}$. The methanol used was from Panreac, UHPLC, 99.9\% purity, degasified previous to deposition.

Infrared spectra were obtained in the IEM-CSIC laboratory at Madrid. Ices of methanol were grown in a high vacuum $150 \mathrm{~mm}$ diameter cylindrical chamber pumped with a $2101 \mathrm{~s}^{-1}$ (for $\mathrm{N}_{2}$ ) turbo pump through a DN100 CF flange placed in its base. The chamber is provided with a closed-cycle He cryostat, coupled to a Fourier transform infrared spectrometer and a $\mathrm{He}-\mathrm{Ne}$ laser to measure ice thickness by interferometry (see Fig. 2). The gas was introduced into the chamber through a needle valve coming from a methanol line (Fisher Scientific, HPLC, $99.99 \%$ purity) thermally stabilized at a vapour pressure of $40 \pm 2$ mbar. This low pressure was achieved and held constant immersing a methanol flask in an ice-water bath. The gas inlet is situated $120 \mathrm{~mm}$ below the optical axis transversal plane, and $200 \mathrm{~mm}$ above the pump flange. The geometry of the chamber was especially designed to obtain a symmetric deposition on both sides of the IR transparent Si wafer, placed in close contact with the cold head of the cryostat. This was confirmed by monitoring with the He-Ne laser the desorption of the back-side deposit. We found that we have the same thickness in both faces, within $5 \%$ error. For the experiments performed in this publication we employed a constant growth rate of $1.2 \mathrm{~nm} \mathrm{~s}^{-1}$, estimated using Eq. (2), and the time it takes to grow an interference maximum. At this growing rate, the water contamination of the methanol ice, due to the residual water vapour in the chamber, is less than $0.1 \%$. The experiments were performed via the following sequence: first, the desired temperature was set; then gas was introduced in the chamber while $\mathrm{He}-\mathrm{Ne}$ interference fringes were recorded; after one maximum of interference was grown, the gas inlet valve was closed, and a MIR spectrum was taken. 


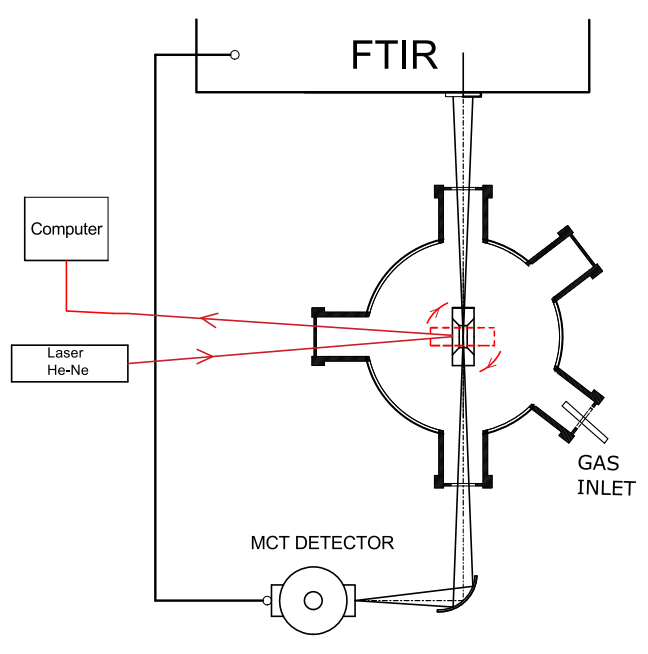

Fig. 2. Scheme of the experimental set-up used to record transmission IR spectra of methanol ices.

This sequence was repeated until four interference maxima were grown and four MIR spectra were obtained. We note that it was necessary to rotate alternatively the $\mathrm{Si}$ substrate to face two different windows in the chamber to record $\mathrm{He}-\mathrm{Ne}$ interferences in quasi normal incidence $\left(4.0 \pm 0.2^{\circ}\right)$ or to take FTIR spectra in normal transmission configuration (see Fig. 2). Due to the weakness of the NIR absorptions, no NIR spectra were recorded for those thin layers. Subsequently, at the same temperature, ten more interference maxima were grown and, finally, a near infrared spectrum of the thick ice layer was obtained. Infrared spectra were recorded with a Burker Vertex 70 spectrometer, using a Globar source and a $\mathrm{KBr}$ beam splitter for the MIR spectral range, and a $\mathrm{CaF}$ beamspliter and a NIR lamp for the NIR range. The same mercury cadmium telluride detector was used for both regions. We worked at $2 \mathrm{~cm}^{-1}$ resolution and an accumulation of 300 scans. In these experiments, ice layer thicknesses were determined from $\mathrm{He}-\mathrm{Ne}$ laser interference measurements, using Eq. (2) and $n_{0}$ from Table 1 . To avoid saturation of the MIR absorption bands, only ice layers up to $\approx 1000 \mathrm{~nm}$ thick were considered. In the NIR spectral range, ice layers of about $3400 \mathrm{~nm}$ were employed.

\subsection{Infrared optical constants}

Infrared optical constants and refractive index are denoted in the following $n(\mathrm{NIR})+i k(\mathrm{NIR})$ and $n(\mathrm{MIR})+i k(\mathrm{MIR})$ respectively for the NIR and MIR spectral ranges. In this work we set the limits for the NIR region from $5000(2.0 \mu \mathrm{m})$ to $3600 \mathrm{~cm}^{-1}(2.7 \mu \mathrm{m})$, and for the MIR region from $3600(2.7 \mu \mathrm{m})$ to $650 \mathrm{~cm}^{-1}(15.4 \mu \mathrm{m})$. To determine $n(\mathrm{MIR})$ and $k(\mathrm{MIR})$ we have followed a procedure developed in our group and described in detail in Zanchet et al. (2013). This procedure takes advantage of the redundancy of information available when a set of spectra of different thicknesses of the same ice is available. In particular, four mid-IR spectra with thickness between $200 \mathrm{~nm}$ and $1000 \mathrm{~nm}$ were recorded for each ice. Theoretical transmission spectra were obtained using the Fresnel equations for a multilayer system vacuum/ice/silicon/ice/vacuum, and taking initial input values for $n$ (MIR), $k(\mathrm{MIR}), n_{0}$ (visible refractive index), and $L$ (ice layer thickness). Then the difference between experimental and theoretical spectra for the four experimental spectra available were minimized (see details in Zanchet et al. 2013). In the minimization procedure the measured $n_{0}$ and layer
Table 1. Refractive index at $633 \mathrm{~nm}$ and densities of pure $\mathrm{CH}_{3} \mathrm{OH}$ ices grown at the indicated temperatures.

\begin{tabular}{ccc}
\hline \hline $\begin{array}{c}T \\
(\mathrm{~K})\end{array}$ & $n_{0}$ & $\begin{array}{c}\text { Density } \\
\left(\mathrm{g} \mathrm{cm}^{-3}\right)\end{array}$ \\
\hline 20 & 1.257 & 0.636 \\
40 & 1.273 & 0.664 \\
60 & 1.271 & 0.685 \\
80 & 1.303 & 0.724 \\
100 & 1.343 & 0.798 \\
110 & 1.346 & 0.822 \\
120 & 1.339 & 0.795 \\
130 & 1.346 & 0.838 \\
\hline
\end{tabular}

thickness can vary within experimental error. Additionally, a coherence parameter that varies between 1 and 0 is fitted to consider coherence losses of the IR radiation in its transmission path through the layered system. At the end of the fitting procedure a set of $n(\mathrm{MIR})$ and $k(\mathrm{MIR})$ optical constants is obtained.

For the near infrared spectral range, infrared scattering and coherence losses become important in relation to the intensity of the absorptions, and the model described above, based on Fresnel equations, is not able to reproduce the experimental infrared interference pattern in the $5000-3500 \mathrm{~cm}^{-1}$ region. For this reason, we decided to record a single NIR spectrum for each ice temperature, corresponding to a thickness of about $3400 \mathrm{~nm}$, and to extract $k(\mathrm{NIR})$ applying Lambert-Beer's law

$k(v)=\frac{1}{4 \pi v L} \operatorname{Abs}(v)$,

where $\operatorname{Abs}(v)$ is the absorbance spectrum (baseline subtracted), $L$ the ice thickness, and $v$ the frequency. The real component $n$ (NIR) was then obtained by means of the Kramers-Kroning relation between $n$ and $k$, considering the information we have for $n_{0}, n(\mathrm{MIR})$, and $k(\mathrm{MIR})$.

\subsection{Band strengths}

The integrated band strengths can be obtained from the imaginary part of the refractive index via

$A=\frac{4 \pi}{\rho} \int_{\text {band }} v k(v) \mathrm{d} v$,

where $\rho$ is the ice density. Ice densities at different temperatures were taken from Table 1 . When the imaginary part of the refractive index is not known it is possible to obtain an effective band strength, $A^{\prime}$, from the absorbance spectrum and the column density of absorbing species, using

$A^{\prime}=\frac{2.303}{\rho L} \int_{\text {band }} \operatorname{Abs}(v) \mathrm{d} v$.

The effective band strength can be affected by scattering and IR interferences that disturb the baseline of the absorption spectra and are difficult to disentangle form real absorptions (Hudson et al. 2014). It is interesting to point out that Eqs. (5) and (6) both involve the density of the ice. Ice densities are usually unknown and an $A^{\prime}$ value is usually estimated assuming an ice density of $1 \mathrm{~g} \mathrm{~cm}^{-3}$. This was not the case in the present work, where we have measured the densities of the ices in an independent experiment (see Table 1). 


\section{Results}

\subsection{Densities and refractive indices.}

Figure 3 collects density and refractive indices at eight temperatures covering the range from 20 to $130 \mathrm{~K}$. Each experimental point is the result of two experiments except for $100 \mathrm{~K}$, which corresponds to the mean value of eight different experiments. Experimental errors are calculated from the $t$-student with $95 \%$ confidence, from 12 experiments for $T \geq 100 \mathrm{~K}$, and applied to the complete data set. This procedure gives errors of $0.01 \mathrm{~g} \mathrm{~cm}^{-3}$ for densities and 0.01 for the refractive indices.

The two parameters have the same general behaviour: $\rho$ and $n_{0}$ grow with the temperature of deposition from $20 \mathrm{~K}$ to $100 \mathrm{~K}$. For higher temperatures both remain almost constant. The lower values correspond to $0.64 \mathrm{~g} \mathrm{~cm}^{-3}$ and 1.26 at $20 \mathrm{~K}$, and the higher values to $0.813 \mathrm{~g} \mathrm{~cm}^{-3}$ and 1.344 for $T \geq 100 \mathrm{~K}$. In the density plot we have indicated the value used in the bibliography for methanol, $1 \mathrm{~g} \mathrm{~cm}^{-3}$ (Hudgins et al. 1993; Maté et al. 2009; Bouilloud et al. 2015; Torrie et al. 1989).

Refractive indices vary from about 1.26 to 1.34 . This last value is almost constant for $T \geq 100 \mathrm{~K}$, matching the refractive index usually employed in the bibliography, 1.33 (Hudgins et al. 1993; Bouilloud et al. 2015), but disagreeing for lower temperatures of deposition (from 20 to $80 \mathrm{~K}$ ). Our experimental refractive indices match those obtained by Isokoski et al. (2014; blue squares in Fig. 3). Both sets of experiments show increasing $n_{0}$ values from $20 \mathrm{~K}$ to $100 \mathrm{~K}$. In both cases the slope seems to vary depending on the deposition temperature. The lowest slope corresponds to temperatures ranging from 40 to $60 \mathrm{~K}$. This seems to be similar to the values reported in Cazaux et al. (2015) for water ice simulations, and would be related to the number of neighbours a molecule is interacting with.

This overall behaviour is similar to that of other molecules studied before: $\mathrm{CO}_{2}$ (Satorre et al. 2008), $\mathrm{NH}_{3}$ (Satorre et al. 2013), and $\mathrm{C}_{2} \mathrm{H}_{4}$ and $\mathrm{C}_{2} \mathrm{H}_{6}$ (Satorre et al. 2017). An amorphous structure is formed at low temperature that increases its order with increasing deposition temperature until a maximum density (or refractive index) is achieved, corresponding to a crystalline structure. Methanol is atypical because this maximum (at $100 \mathrm{~K}$ ) does not imply that the crystal is formed, as indicated by the IR spectra. These spectra reveal different crystalline structures at $T \geq 110 \mathrm{~K}$, but its refractive indices and densities remain almost constant. The small variations in these values in Fig. 3 above $100 \mathrm{~K}$ are difficult to assign to structural changes within our error bars.

\subsection{Infrared spectra}

Figure 4 shows an example of the spectra obtained for methanol ices grown at eight different temperatures, from $20 \mathrm{~K}$ to $130 \mathrm{~K}$. An adequate baseline has been subtracted. An amorphous solid is formed in deposits at temperatures equal to or below $100 \mathrm{~K}$. At these temperatures the spectra have a similar shape, denoting the amorphous structure, although some differences can be appreciated. There is an overall tendency for all the bands to become narrower and to increase their maximum peak intensity values with increasing deposition temperature (see e.g. the band at $1027 \mathrm{~cm}^{-1}$ ). Interestingly, at higher wavenumbers the broad $\mathrm{OH}$ stretching band at $3250 \mathrm{~cm}^{-1}$ presents a shoulder for ices grown between 20 and $60 \mathrm{~K}$ which disappears at $80 \mathrm{~K}$. The band is amplified in Fig. 5a. The presence of that shoulder at $3400 \mathrm{~cm}^{-1}$ might be correlated with the density of the ice (see Fig. 3) the spectral signature being larger for lower ice
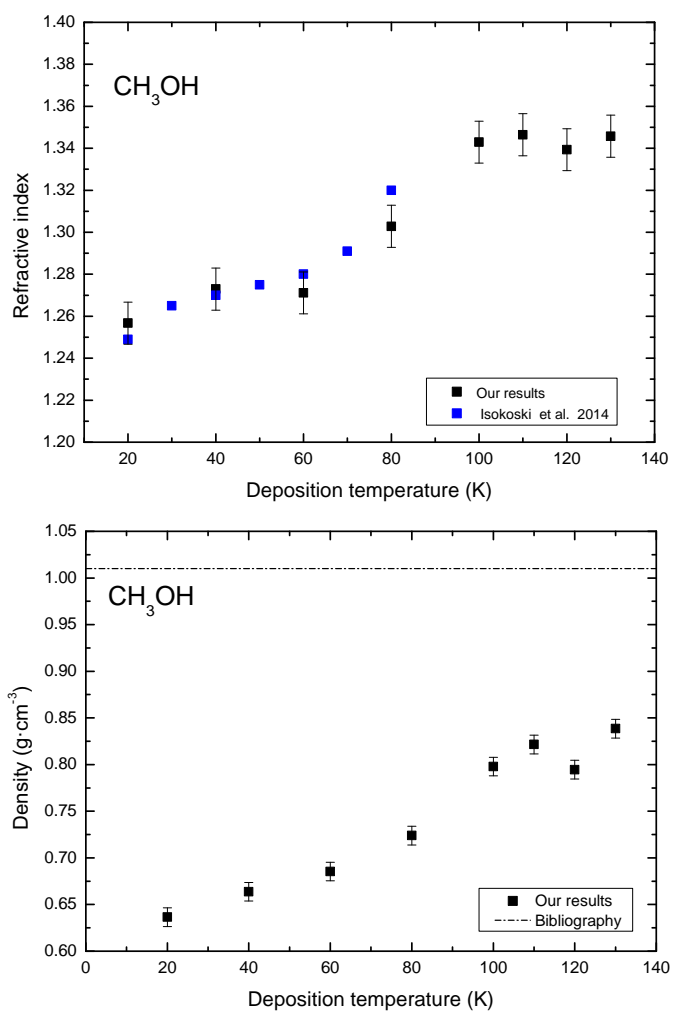

Fig. 3. Variation in density and refractive index of methanol with deposition temperature.

densities. A possible interpretation of these results, in connection with what is observed for water ice, could be the presence of pores in the amorphous methanol ice where methanol molecules are situated with weakly bonded $\mathrm{O}-\mathrm{H}$ bond or even dangling bonds responsible of the larger wavenumber absorptions in the spectrum.

Between $100 \mathrm{~K}$ and $110 \mathrm{~K}$ a phase transition from amorphous to polycrystalline solid is revealed by the strong changes that are observed in the IR spectra (see Fig. 5b). However, the crystalline form obtained at $110 \mathrm{~K}$ changes when the ice is deposited at $120 \mathrm{~K}$ and above. The intermediate phase at $110 \mathrm{~K}$ could be a different crystalline form or maybe just a mixture of amorphous and crystalline phases. Nonetheless, taking a closer look to the spectrum of the ice at $110 \mathrm{~K}$, it is observed that there are already very sharp features at $4000 \mathrm{~cm}^{-1}, 2950 \mathrm{~cm}^{-1}$, and $1450 \mathrm{~cm}^{-1}$, pointing to the presence of a crystalline form at $110 \mathrm{~K}$. It is interesting to note here the result by Isokoski et al. (2014) that follows the interference signal of a He-Ne laser during thermal annealing of methanol ice grown at $20 \mathrm{~K}$. They found an abrupt decay of the signal at about $100 \mathrm{~K}$, which denotes a compaction of the ice before crystallization. This process of compaction but not crystallization agrees with what is observed in our experiments (see Figs. 3-5).

We have also followed the infrared spectral changes of an ice upon annealing at $5 \mathrm{~K} \mathrm{~min}^{-1}$ from $20 \mathrm{~K}$ to $130 \mathrm{~K}$. Figure 6 shows two absorption regions chosen to illustrate the differences in the spectra due to the different degrees of crystallization obtained by direct deposition (left panel) or thermal annealing (right panel). At $110 \mathrm{~K}, 120 \mathrm{~K}$, and $130 \mathrm{~K}$ the crystalline ice obtained by annealing does not reach the degree of crystallinity that the ices grown by direct vapour deposition have at thdse same temperatures. 

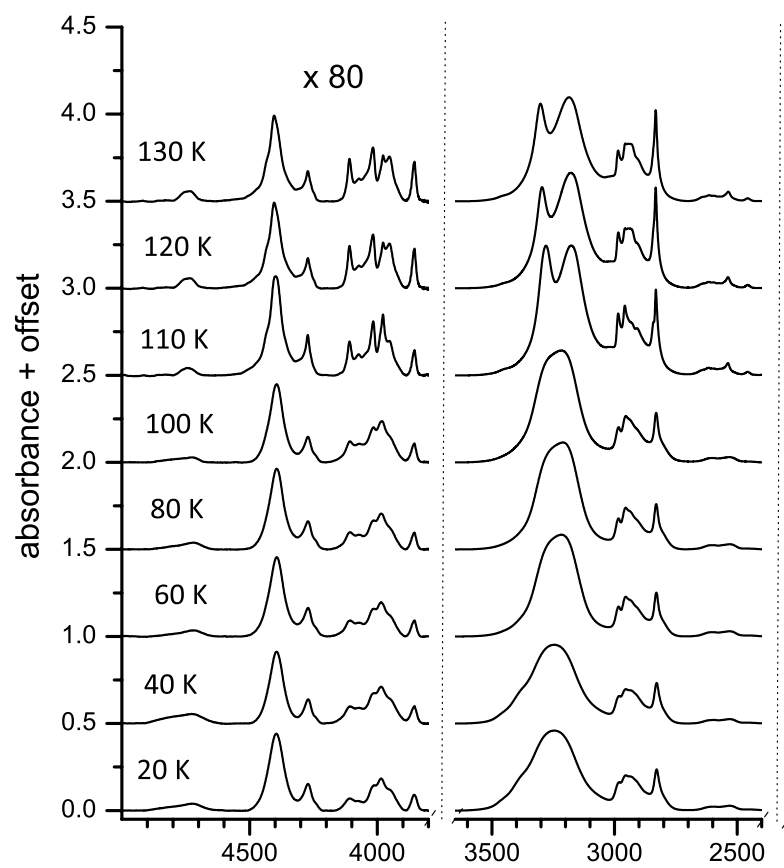

wavenumber $\mathrm{cm}^{-1}$
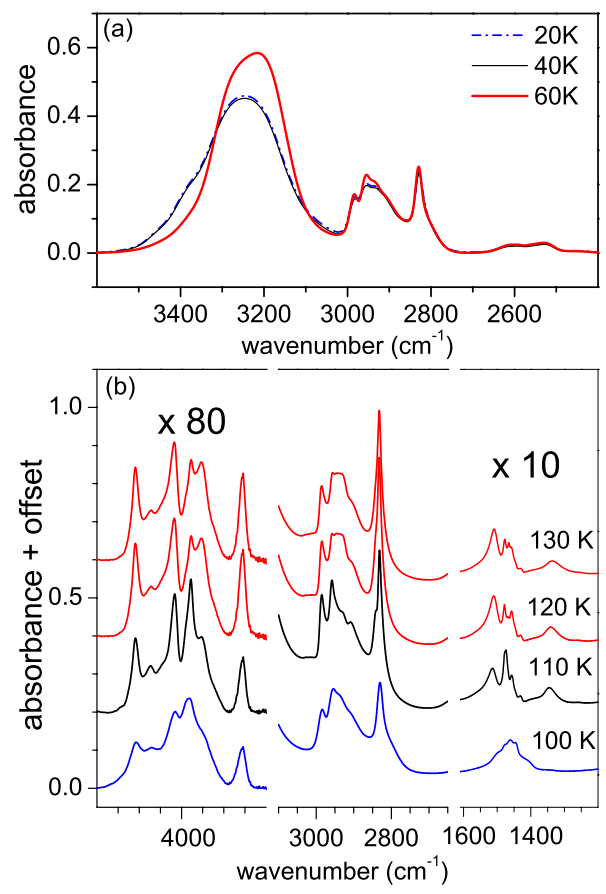

Fig. 5. Enlargement of part of the spectra presented in Fig. 4 to highlight (panel a) the shoulder at higher wavenumbers in the $3250 \mathrm{~cm}^{-1}$ band, and (panel $b$ ) the differences between compact-amorphous $(100 \mathrm{~K})$ and crystalline structures $(110 \mathrm{~K}, 120 \mathrm{~K}, 130 \mathrm{~K})$.

\subsection{Optical constants and band strengths}

The NIR and MIR optical constants of solid methanol obtained in this work by the procedure described in Sect. 2.1 are presented in Fig. 7. It is important to remember that for the determination of these magnitudes from the IR spectra it is not necessary to know the ice densities, only the thickness of the ice layers. At the end of the iterative procedure, the simulated spectra reproduce the experimental observations with a root mean square deviation

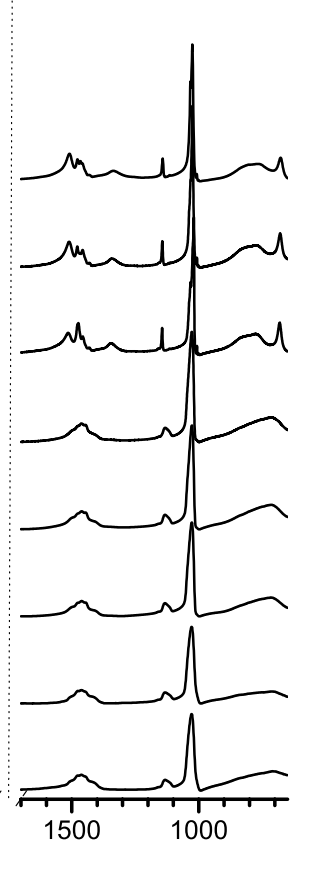

Fig. 4. Infrared spectra of methanol ices grown at the indicated temperatures. Appropriate baselines have been subtracted. The spectra correspond to layers about $990 \mathrm{~nm}$ thick. Spectral regions with weak absorptions have been multiplied by a factor indicated in the figure. Vertical offsets have been added to the absorbance spectra for clarity in the representation.
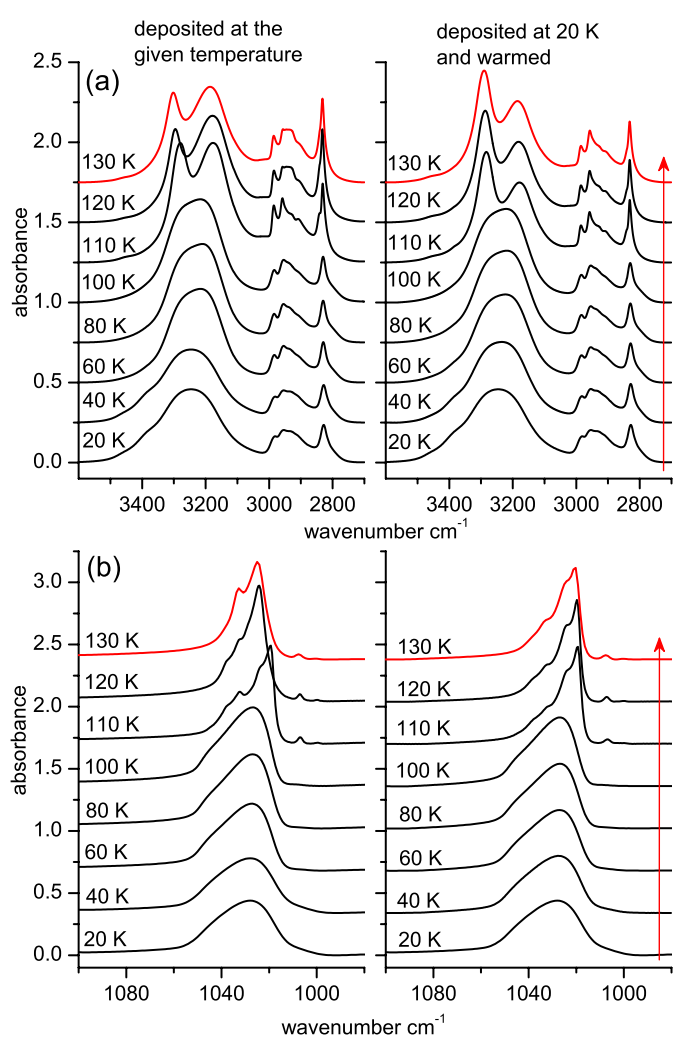

Fig. 6. Infrared spectra of methanol ices grown at the indicated temperatures (left panels) or grown at $20 \mathrm{~K}$ and annealed to the indicated temperatures (right panels). Appropriate baselines have been subtracted. The spectra correspond to layers about $990 \mathrm{~nm}$ thick. Vertical offsets have been added to the absorbance spectra for clarity in the representation.

of 0.001 in absorbance units. We estimate overall uncertainties of $\approx 1 \%$ for the real component of the refractive index $n$. The uncertainty is also $\approx 1 \%$ for the imaginary component, $k$, except 


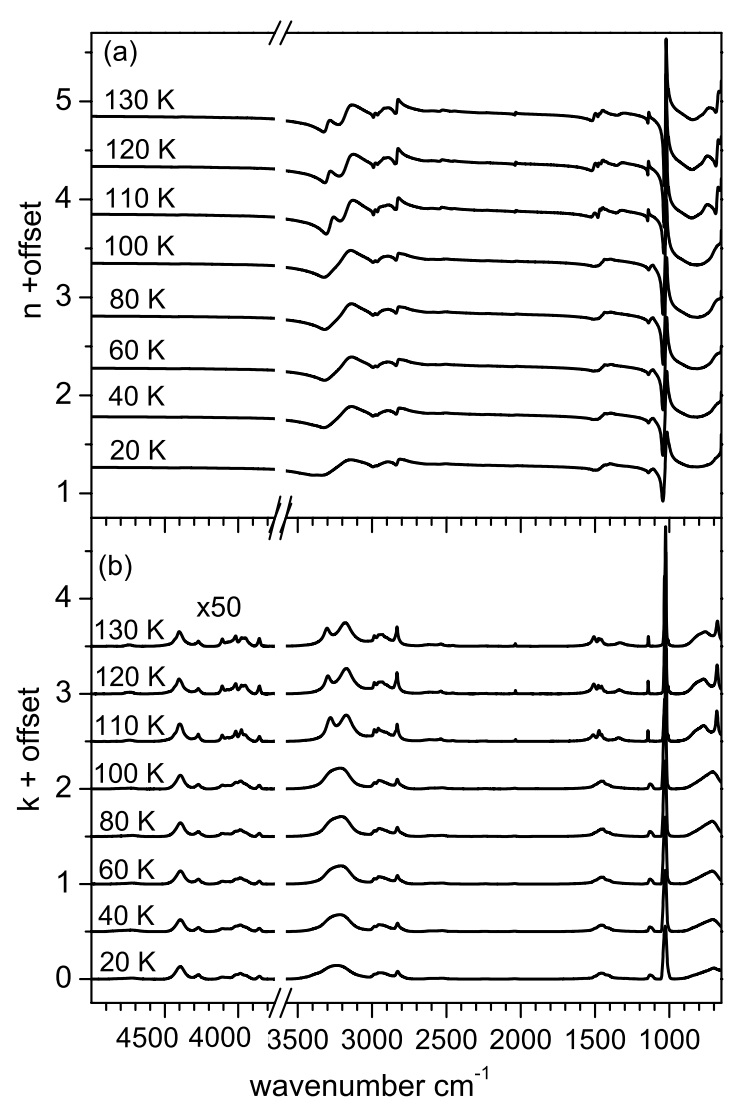

Fig. 7. Infrared real $(n)$ and imaginary $(k)$ part of the refractive index of methanol ices at different deposition temperatures. Vertical offsets have been added to the magnitudes for clarity in the representation. In the lower panel, $k$ multiplied by 50 has been represented in the 3700 and $5000 \mathrm{~cm}^{-1}$ spectral range.

when $k<0.01$, which induces larger errors due to the higher signal-to-noise ratio in the film-substrate transmittance.

In Tables 2 and 3 we present the NIR and MIR band strengths obtained in this work at different temperatures for amorphous and crystalline ices, respectively. Mode assignments are presented in the first two columns. In the third column peak positions are listed, although for most cases several unresolved peaks are present in the integration region, and these positions are somewhat arbitrary. The fourth column indicates the integration intervals employed. We have estimated the absolute band strengths, $A$, and the effective band strengths, $A^{\prime}$, in the MIR region using the expressions in Eqs. (5) and (6), respectively. In the NIR spectral range the two magnitudes coincide since the $k$ values were obtained after subtracting a baseline and applying the Lambert-Beer law. The ice densities needed were taken from Table 1. An average experimental error of $20 \%$ was estimated for theses magnitudes, being smaller for the stronger features and larger for the weaker ones. This error is mostly due to the criteria used to choose the integration intervals.

In Fig. 8 we have represented the evolution of the band strengths with temperature for two bands. It can be seen that the band strengths do not change appreciably, within experimental error, for temperatures below $100 \mathrm{~K}$. However, some modifications are observed when the ice phase changes from amorphous to crystalline above $100 \mathrm{~K}$. These findings are in good agreement with those of Kerkhof et al. (1999), where no variation was found for the band strength of pure methanol ices grown at $10 \mathrm{~K}$ and annealed up to $100 \mathrm{~K}$. In that work, higher temperatures were not investigated for the pure species. At higher temperatures, the observed changes point to the different crystalline structures formed by deposition at $110 \mathrm{~K}, 120 \mathrm{~K}$, or $130 \mathrm{~K}$.

\section{Discussion}

To our knowledge the article Hudgins et al. (1993; hereafter HSAT) presents the most complete set of IR data of pure methanol ice published so far. In particular, they provide the only set of optical constants reported for pure solid methanol in the MIR range. These authors studied a methanol ice grown at $10 \mathrm{~K}$ and annealed to higher temperatures. In Fig. 9 we compare the optical constants we obtained for methanol ice at $20 \mathrm{~K}$ to those by HSAT at $10 \mathrm{~K}$. It can be seen that the literature values are higher and they approach our data if they are scaled by a factor 0.64 . This factor is close to the density we measured for methanol ice at low temperature (see Table 1).

Although the imaginary part of the refractive index, $k$, as a characteristic of the solid depends on its density, it can be determined without the knowledge of that magnitude. The only ice parameter needed for calculating $k$ is the visible refractive index of the ice, $n_{0}$, which is needed to obtain the ice layer thickness from Eq. (2). The refractive index assumed in HSAT for methanol ice was 1.33 , taken from the value for liquid $\mathrm{CH}_{3} \mathrm{OH}$ given by Weast (1972). The refractive index we have measured for methanol at $20 \mathrm{~K}$ in this work is 1.26 . From this data, the ice film thickness considered by HSAT could be underestimated. However, it is likely that the ice grown by HSAT at $10 \mathrm{~K}$ will not have the same $n_{0}$ or density as the ice grown at $20 \mathrm{~K}$ in our work. In their experiments the gas was introduced into the vacuum chamber though a tube directed perpendicular to the cold substrate, while in our work the whole vacuum chamber was filled with a homogenous pressure of methanol gas (background deposition). Random-trajectory background deposition generates more porous ices than directional deposition (Dohnálek et al. 2003; Bossa et al. 2015). Therefore, it is expected that the methanol ice grown by HSAT at $10 \mathrm{~K}$ is more compact than the ice grown in the present work at $20 \mathrm{~K}$. A smaller number of absorbers, related to the large porosity of our samples, will be evidenced as a decrease in the imaginary part of the refractive index. On the contrary, a more compact ice will absorb more IR radiation, which means higher $k$ values. These considerations are in agreement with the scaling factor applied to match the two sets of $k$ values represented in Fig. 9. Apart from the overall scaling factor, differences in the shape and relative intensities of some features of the two sets of optical constants can be seen, mostly above $100 \mathrm{~K}$. In Fig. 10 the $k$ values given by HSAT at different temperatures are compared to our data for the bands at $\approx 2833 \mathrm{~cm}^{-1}(3.53 \mu \mathrm{m})$ and at $\approx 1027 \mathrm{~cm}^{-1}(9.74 \mu \mathrm{m})$, two of the peaks most widely employed for methanol identification in astrophysical media. The literature data in Fig. 10 have been scaled for clarity. There is very good agreement in the shape of the two sets of constants for amorphous samples from $10 \mathrm{~K}$ up to $100 \mathrm{~K}$ (see the four lower lines in both panels). However, the differences in shape are significant between the $k$ values reported in HSAT for crystalline ice at $120 \mathrm{~K}$ and the three sets of constants at $110 \mathrm{~K}, 120 \mathrm{~K}$, and $130 \mathrm{~K}$ reported in this work (see the four upper lines in both panels). This situation shows that the structure obtained by direct deposition is more ordered than that obtained by an ice deposited at low temperature and annealed.

Different band strengths for $\mathrm{CH}_{3} \mathrm{OH}$ ice at low temperature reported in the literature are compared with our data in Table 4. The $A^{\prime}$ estimated by HSAT assuming an ice density of $1 \mathrm{~g} \mathrm{~cm}^{-3}$ coincides with our data. As commented above, we believe HSAT 
R. Luna et al.: Densities, infrared band strengths, and optical constants of solid methanol

Table 2. Absolute $(A)$ and effective $\left(A^{\prime}\right)$ band strengths of $\mathrm{CH}_{3} \mathrm{OH}$ amorphous ice grown at the indicated temperatures.

\begin{tabular}{|c|c|c|c|c|c|c|c|c|c|c|c|c|c|}
\hline \multirow[t]{7}{*}{ Label } & \multirow[t]{7}{*}{ Mode } & \multirow{3}{*}{$\begin{array}{l}\text { Peak } \\
\mathrm{cm}^{-1}\end{array}$} & \multirow{3}{*}{$\begin{array}{c}\begin{array}{c}\text { Integration } \\
\text { interval } \\
\mathrm{cm}^{-1}\end{array} \\
4908-4649\end{array}$} & \multirow{2}{*}{\multicolumn{2}{|c|}{$\begin{array}{l}A \quad A^{\prime} \\
100 \mathrm{~K} \\
\end{array}$}} & \multirow[t]{2}{*}{$A$} & \multirow{2}{*}{$\mathrm{K}^{A^{\prime}}$} & \multirow{2}{*}{$\begin{array}{l}A \\
{ }^{18} \mathrm{~cm} \\
6 \\
6\end{array}$} & \multirow{2}{*}{$\begin{array}{l}A^{\prime} \\
\text { nolecul } \\
\mathrm{K} \\
\end{array}$} & \multirow{2}{*}{\multicolumn{2}{|c|}{$\begin{array}{l}\text { A } \\
-1) \\
\end{array}$}} & \multirow{2}{*}{\multicolumn{2}{|c|}{$\begin{array}{l}A \quad A^{\prime} \\
20 \mathrm{~K}\end{array}$}} \\
\hline & & & & & & & & & & & & & \\
\hline & & & & - & 0.039 & - & 0.053 & - & 0.05 & - & 0.092 & - & 0.028 \\
\hline & & 4395 & $4530-4201$ & - & 0.417 & - & 0.494 & - & 0.508 & - & 0.477 & - & 0.578 \\
\hline & & 4271 & $4316-4201$ & - & 0.046 & - & 0.053 & - & 0.056 & - & 0.053 & - & 0.058 \\
\hline & & 3983 & $4201-3886$ & - & 0.339 & - & 0.31 & - & 0.305 & - & 0.337 & - & 0.289 \\
\hline & & 3856 & $3888-3821$ & - & 0.028 & - & 0.029 & - & 0.029 & - & 0.031 & - & 0.034 \\
\hline$v_{1}$ & $\mathrm{OH}$ str. & & & & & & & & & & & & \\
\hline$v_{3}, v_{9}$ & $\mathrm{CH}_{3}$ str. & 2950 & $3600-2700$ & 153 & 145 & 159 & 148 & 155 & 146 & 156 & 150 & 151 & 142 \\
\hline$v_{3}, v_{9}$ & $\mathrm{CH}_{3}$ str. & 2830 & $2865-2750$ & 4.51 & 4.48 & 4.88 & 4.74 & 4.89 & 4.72 & 4.06 & 4.97 & 5.06 & 4.86 \\
\hline & & 2570 & $2710-2400$ & 2.92 & 2.75 & 3.18 & 2.95 & 3.11 & 2.93 & 3.09 & 2.99 & 2.93 & 2.74 \\
\hline & & 2240 & $2300-2204$ & 0.22 & 0.2 & 0.24 & 0.24 & 0.24 & 0.24 & 0.24 & 0.24 & 0.22 & 0.22 \\
\hline & & 2043 & $2105-2014$ & 0.31 & 0.32 & 0.33 & 0.33 & 0.31 & 0.31 & 0.31 & 0.3 & 0.27 & 0.24 \\
\hline $\begin{array}{l}v_{4}, v_{5}, \\
v_{6}, v_{10}\end{array}$ & $\begin{array}{l}\mathrm{CH}_{3} \text { bend } \\
\mathrm{OH} \text { bend }\end{array}$ & 1460 & $1655-1302$ & 11 & 8.91 & 11.7 & 9.39 & 11.3 & 9.01 & 11 & 9.06 & 11 & 8.05 \\
\hline$v_{7}$ & $\mathrm{CH}_{3}$ rock & 1126 & $1178-1103$ & 1.39 & 1.17 & 1.57 & 1.33 & 1.59 & 1.32 & 1.65 & 1.33 & 1.61 & 1.35 \\
\hline$v_{8}$ & $\mathrm{CO}$ str. & 1027 & 1103-999 & 15.9 & 16.9 & 16.9 & 17.4 & 16.4 & 17 & 16.4 & 17.7 & 16.1 & 15.6 \\
\hline$v_{12}$ & Torsion & 678 & $992-639$ & 19 & 19.4 & 19.2 & 23.2 & 18.3 & 18.6 & 19.3 & 15.1 & 18.1 & 22.9 \\
\hline
\end{tabular}

Notes. First two columns: mode assignment (Gálvez et al. 2009); Third column: wavenumber of the strongest peak in the integration interval; Fourth column: integration interval.

Table 3. Absolute $(A)$ and effective $\left(A^{\prime}\right)$ band strengths of $\mathrm{CH}_{3} \mathrm{OH}$ crystalline ice grown at the indicated temperatures.

\begin{tabular}{|c|c|c|c|c|c|c|c|c|c|}
\hline \multirow[t]{2}{*}{ Label } & \multirow[t]{2}{*}{ Mode } & \multirow[t]{2}{*}{$\begin{array}{l}\text { Peak } \\
\mathrm{cm}^{-1}\end{array}$} & \multirow{2}{*}{$\begin{array}{c}\text { Integration } \\
\text { interval } \\
\mathrm{cm}^{-1}\end{array}$} & \multicolumn{6}{|c|}{$\left(\times 10^{18} \mathrm{~cm}\right.$ molecule $\left.{ }^{-1}\right)$} \\
\hline & & & & \multicolumn{2}{|c|}{$130 \mathrm{~K}$} & \multicolumn{2}{|c|}{$120 \mathrm{~K}$} & \multicolumn{2}{|c|}{$110 \mathrm{~K}$} \\
\hline & & 4742 & $4812-4649$ & - & 0.031 & - & 0.041 & & 0.028 \\
\hline & & 4399 & $4622-4200$ & - & 0.44 & - & 0.469 & & 0.527 \\
\hline & & 4270 & $4322-4224$ & - & 0.045 & - & 0.049 & & 0.058 \\
\hline & & 4026 & $4168-3888$ & - & 0.411 & - & 0.436 & & 0.402 \\
\hline & & 3858 & $3888-3823$ & - & 0.049 & - & 0.054 & & 0.033 \\
\hline $\begin{array}{c}v_{1} \\
v_{3}, v_{9}\end{array}$ & $\begin{array}{l}\mathrm{OH} \text { str. } \\
\mathrm{CH}_{3} \text { str. }\end{array}$ & 2950 & $3600-2700$ & 164 & 150 & 179 & 168 & 185 & 178 \\
\hline \multirow[t]{4}{*}{$v_{3}, v_{9}$} & $\mathrm{CH}_{3}$ str. & 2832 & $2875-2785$ & 7.03 & 6.51 & 8.15 & 7.62 & 6.24 & 6.08 \\
\hline & & 2537 & $2704-2413$ & 4.21 & 3.9 & 4.56 & 4.25 & 4.5 & 4.47 \\
\hline & & 2251 & $2302-2206$ & 0.29 & 0.28 & 0.33 & 0.3 & 0.32 & 0.33 \\
\hline & & 2036 & $2077-2020$ & 0.35 & 0.38 & 0.38 & 0.42 & 0.26 & 0.29 \\
\hline$v_{4}, v_{5}$ & $\mathrm{CH}_{3}$ bend & & & & & & & & \\
\hline \multirow[t]{2}{*}{$v_{6}, v_{10}$} & $\mathrm{OH}$ bend & 1460 & $1742-1420$ & 7.24 & 8.12 & 8.11 & 9.26 & 6.97 & 8.62 \\
\hline & & 1336 & $1421-1235$ & 2.09 & 1.87 & 2.03 & 1.94 & 1.57 & 1.81 \\
\hline & $\mathrm{CH}_{3}$ rock & 1142 & $1224-1132$ & 0.68 & 0.72 & 0.76 & 0.85 & 0.61 & 0.73 \\
\hline$v_{8}$ & CO str. & 1027 & 1133-996 & 12.8 & 11.7 & 14.5 & 13.1 & 11.8 & 11.7 \\
\hline$v_{12}$ & torsion & 720 & $991-650$ & 18.9 & 15.1 & 20.3 & 22.4 & 20.7 & 19.2 \\
\hline
\end{tabular}

Notes. First two columns: mode assignment; third column: wavenumber of the strongest peak in the integration interval; fourth column: integration interval.

methanol ice is denser than that grown in this work, and its density could be close to $1 \mathrm{~g} \mathrm{~cm}^{-3}$. It could be the case that the actual density of their ice is lower. The agreement found could then be explained by an underestimation of their ice layer thickness values, since this would lead to a similar number of absorbers (molecules $\mathrm{cm}^{-2}$ ). It can be seen in the fourth column of Table 4 that correcting HSAT $A^{\prime}$ values considering the methanol ice density measured in this work, $0.64 \mathrm{~g} \mathrm{~cm}^{-3}$ at $20 \mathrm{~K}$, leads to a large disagreement. In the last column of Table 4 the results of a recent work by Bouilloud et al. (2015) are presented. 


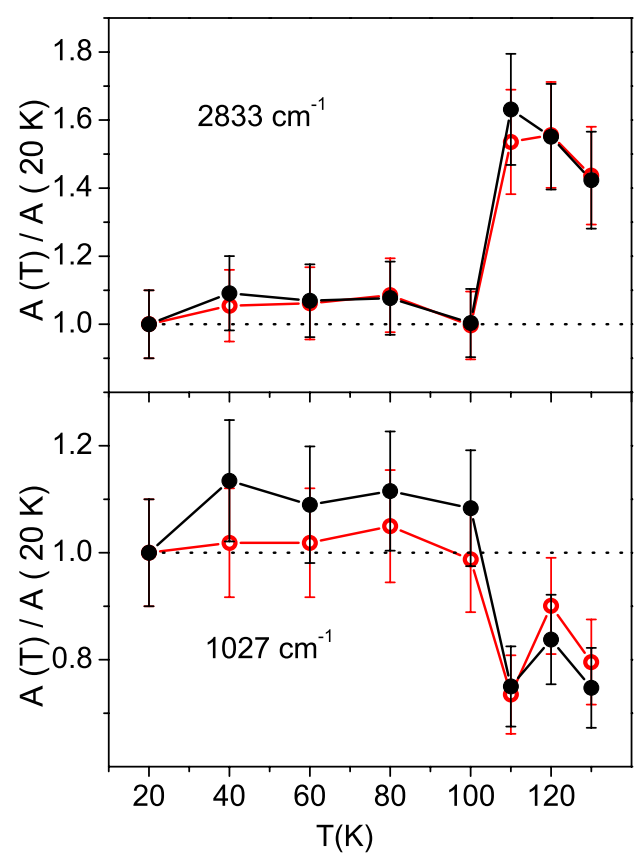

Fig. 8. Temperature dependence of methanol IR band strengths at $2833 \mathrm{~cm}^{-1}$ and $1027 \mathrm{~cm}^{-1}$. The two sets of data represented correspond to $A$ (open red circles) and $A^{\prime}$ (solid black circles).

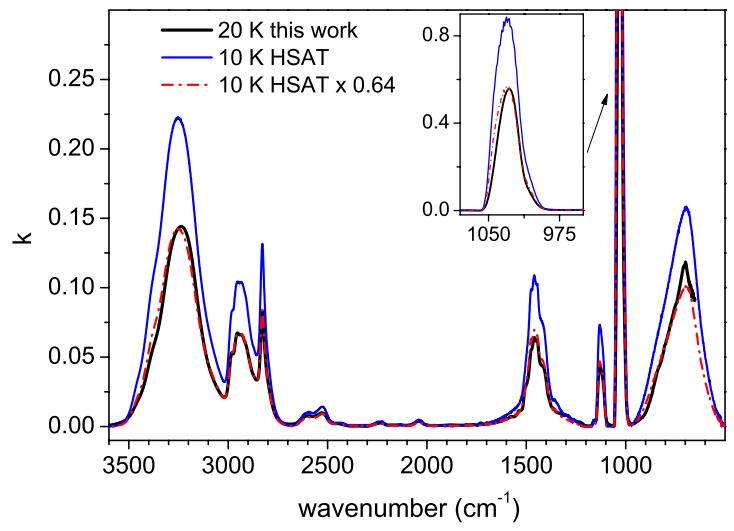

Fig. 9. Comparison of the imaginary part of the refractive index of solid methanol ice at $20 \mathrm{~K}$ provided in this work (black) with the values reported by Hudgins et al. (1993; blue). The scaled literature values are represented with a dashed red line.

They correspond to band strengths of methanol ice grown at $25 \mathrm{~K}$ by deposition through a capillary that is $10 \mathrm{~cm}$ away from the cold surface, with an incidence angle of $45^{\circ}$. This could be considered similar to background deposition and the ice could have low density, $0.64 \mathrm{~g} \mathrm{~cm}^{-3}$, like those grown in the present work at $20 \mathrm{~K}$. When the values presented in Bouilloud et al. (2015) are corrected assuming an ice density of $0.64 \mathrm{~g} \mathrm{~cm}^{-3}$, they coincide very well with our data.

\section{Conclusions}

We have measured new densities, $\rho$, visible refractive indices at $633 \mathrm{~nm}, n_{0}$, IR optical constants, and IR band strengths for methanol ices grown by vapour deposition at $20 \mathrm{~K}, 40 \mathrm{~K}, 60 \mathrm{~K}$, $80 \mathrm{~K}, 100 \mathrm{~K}, 110 \mathrm{~K}, 120 \mathrm{~K}$, and $130 \mathrm{~K}$.

Methanol-ice densities and visible refractive indices increase with the temperature of deposition in the interval 20-100 K.

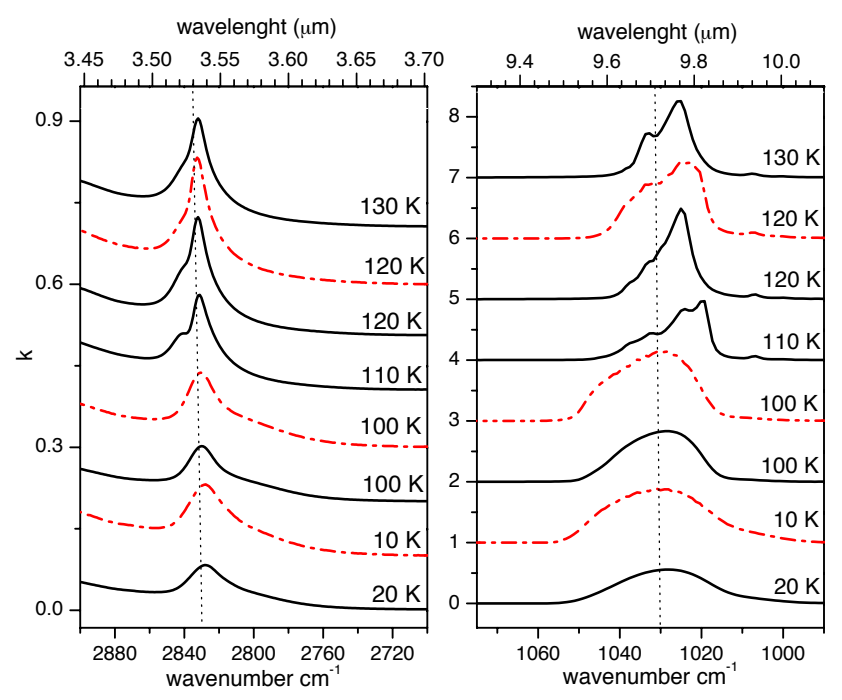

Fig. 10. Comparison between $k$ values from this work (solid black lines) and those from Hudgins et al. (1993; dashed red lines). Literature data have been scaled for clarity in the representation. Vertical offsets have been added to the magnitudes for the same purpose.

They remain almost constant from $100 \mathrm{~K}$ to $130 \mathrm{~K}$, which is the highest temperature investigated. The growth is sharper for the interval $60-100 \mathrm{~K}$, in agreement with the $n_{0}$ values obtained by Isokoski et al. (2014) in similar accretion conditions. The refractive index does not vary between 40 and $60 \mathrm{~K}$, a behaviour that resembles that obtained by Cazaux et al. (2015) for water ices.

Methanol-ice grown at $100 \mathrm{~K}$ presents an anomalous behaviour. It acquires an amorphous structure as dense as the crystalline form. This was revealed by looking at our density and infrared spectra measurements. This particularity has not been observed for any other molecular ice previously studied in our laboratory. For example, $\mathrm{CO}_{2}, \mathrm{C}_{2} \mathrm{H}_{6}, \mathrm{C}_{2} \mathrm{H}_{4}$, and $\mathrm{NH}_{3}$ ices have a lower density in amorphous phases than in crystalline phases. This finding coincides with the sudden ice compaction that takes place at $100 \mathrm{~K}$ for $\mathrm{CH}_{3} \mathrm{OH}$ ice when deposited at $20 \mathrm{~K}$ and warmed up as shown in Isokoski et al. (2014). This compaction is not associated with crystallization either, as shown by infrared spectroscopy when annealing $\mathrm{CH}_{3} \mathrm{OH}$ to $100 \mathrm{~K}$.

Infrared spectra of crystalline methanol obtained by annealing ices grown at $20 \mathrm{~K}$ to $110 \mathrm{~K}, 120 \mathrm{~K}$, and $130 \mathrm{~K}$ show that they are different to the crystalline ices obtained by direct vapour deposition at these temperatures. Optical constants in the NIR and MIR spectral ranges, from $5000 \mathrm{~cm}^{-1}$ to $650 \mathrm{~cm}^{-1}$, are provided for amorphous and crystalline methanol ices grown at eight temperatures from $20 \mathrm{~K}$ to $130 \mathrm{~K}$. These optical constants differ from the Hudgins et al. (1993) data, due to the different ice-generation conditions which lead to different morphologies and phases.

We also present methanol band strengths in the NIR and MIR spectral ranges at the same eight temperatures mentioned above. Some band strengths vary for crystallized ices up to $30 \%$. This temperature dependence must be considered to properly estimate the amount of methanol in different astrophysical environments.

For low temperature band strengths, we have compared our results with previous literature data collected by Bouilloud et al. (2015). We found that at low temperatures $(10 \mathrm{~K}-25 \mathrm{~K})$, methanol band strengths do not depend appreciably on the ice morphology, but only on the number of absorbers. Therefore, all the literature data agree if the adequate ice densities, which depend on the generation conditions, are taken into account. Our 
Table 4. Absolute band strengths $\left(\times 10^{18} \mathrm{~cm} \mathrm{molecule}{ }^{-1}\right)$.

\begin{tabular}{ccccc}
\hline \hline Peak & $\begin{array}{c}\text { This work } \\
\rho=0.64 \\
\left(\mathrm{~g} \mathrm{~cm}^{-3}\right)\end{array}$ & $\begin{array}{c}\text { Hudgins et al. (1993) } \\
\rho=1 \\
\left(\mathrm{~g} \mathrm{~cm}^{-3}\right) \\
10 \mathrm{~K}\end{array}$ & $\begin{array}{c}\text { Hudgins et al. (1993) } \\
\rho=0.64 \\
\left(\mathrm{~g} \mathrm{~cm}^{-3}\right) \\
10 \mathrm{~K}\end{array}$ & $\begin{array}{c}\text { Bouilloud et al. (2015) } \\
\rho=0.64 \\
\left(\mathrm{~g} \mathrm{~cm}^{-3}\right) \\
25 \mathrm{~K}\end{array}$ \\
\hline 2900 & 151 & 160 & 250 & 159 \\
2830 & 5.1 & 5.3 & 8.3 & - \\
2558 & 2.9 & 2.8 & 4.4 & 2.8 \\
1461 & 11 & 12 & 19 & 10 \\
1126 & 1.6 & 1.8 & 2.8 & 2.2 \\
1027 & 16 & 18 & 28 & 17 \\
\hline
\end{tabular}

measurements allowed us to explain discrepancies of about $40 \%$ in some band strengths previously reported by Bouilloud et al. (2015).

The spectral shape of the different features in the infrared spectra of methanol ices are distinctive and can help to trace the phase and temperature of methanol ices detected in the ISM or in different solar system objects, and will give information about the formation history of the ices observed.

Acknowledgements. Funds have been provided for this research by the Spanish MINECO, Project FIS2016-77726-C3-1-P and FIS2016-77726-C3-3-P. Germán Molpeceres acknowledges MINECO PhD grant BES-2014-069355. We are grateful to R. Escribano for helpful discussions. Our skillful technicians C. Santonja, M. A. Moreno, and J. Rodríguez are also gratefully acknowledged.

\section{References}

Boogert, A. C. A., Pontoppidan, K. M., Knez, C., et al. 2008, ApJ, 678, 985

Boogert, A., Gerakines, P., \& Whittet, D. 2015, ARA\&A, 53, 541

Bossa, J.-B., Maté, B., Fransen, C., et al. 2015, ApJ, 814, 47

Bottinelli, S., Boogert, A. C., Bouwman, J., et al. 2010, ApJ, 718, 1100

Bouilloud, M., Fray, N., Bénilan, Y., et al. 2015, MNRAS, 451, 2145

Cazaux, S., Bossa, J.-B., Linnartz, H., \& Tielens, A. G. G. M. 2015, A\&A, 573 A16

Dartois, E., Schutte, W. A., \& Geballe, T. R. 1999, A\&A, 342, L32

d'Hendecourt, L. B., \& Allamandola, L. J. 1986, A\&AS, 64, 453

Dohnálek, Z., Kimmel, G. A., Ayotte, P., Smith, R. S., \& Kay, B. D. 2003 ,

J. Chem. Phys., 118, 364
Drabek-Maunder, E., Greaves, J., Fraser, H. J., Clements, D. L., \& Alconcel, L. N. 2017, Int. J. Astrobiol., DOI: 10.1017/S1473550417000428

Gálvez, O., Maté, B., Martín-Llorente, B., Herrero, V. J., \& Escribano, R. 2009, J. Phys. Chem. A, 113, 3321

Gerakines, P. A., Bray, J. J., Davis, A., \& Richey, C. R. 2005, ApJ, 620, 1140

Hodyss, R., Parkinson, C. D., Johnson, P. V., et al. 2009, Geophys. Res. Lett., 36,2

Hudgins, D. M., Sandford, S. A., Allamandola, L. J., \& Tielens, A. G. G. M. 1993, ApJS, 86, 713

Hudson, R., Ferrante, R., \& Moore, M. 2014, Icarus, 228, 276

Ioppolo, S., van Boheemen, Y., Cuppen, H. M., van Dishoeck, E. F., \& Linnartz, H. 2011, MNRAS, 413, 2281

Isokoski, K., Bossa, J.-B., Triemstra, T., \& Linnartz, H. 2014, Phys. Chem. Chem. Phys., 16, 3456

Kerkhof, O., Schutte, W., \& Ehrenfreund, P. 1999, A\&A, 346, 990

Maté, B., Gálvez, Ó., Herrero, V. J., \& Escribano, R. 2009, ApJ, 690, 486

Merlin, F., Quirico, E., Barucci, M. A., \& de Bergh, C. 2012, A\&A, 544, A20

Molpeceres, G., Satorre, M. A., Ortigoso, J., et al. 2016, ApJ, 825, 156

Öberg, K. I. 2016, Chem. Rev., 116, 9631

Palumbo, M. E., Castorina, A. C., \& Strazzulla, G. 1999, A\&A, 342, 551

Pontoppidan, K. M., Dartois, E., van Dishoeck, E. F., Thi, W.-F., \& D'Hendecourt, L. 2003, A\&A, 404, L17

Sandford, S. A., \& Allamandola, L. J. 1993, ApJ, 417, 815

Satorre, M., Domingo, M., Millán, C., et al. 2008, Plan. Spa. Sci., 56, 1748

Satorre, M., Leliwa-Kopystynski, J., Santonja Molté, C., \& Luna, R. 2013, Icarus, 225, 703

Satorre, M., Millán, C., Molpeceres, G., et al. 2017, Icarus, 296, 179

Sauerbrey, G. 1959, Z. Phys., 155, 206

Torrie, B. H., Weng, S. X., \& Powell, B. M. 1989, Mol. Phys., 67, 575

Watanabe, N., \& Kouchi, A. 2002, ApJ, 571, 173

Weast, R. 1972, Handbook of Chemistry and Physics, 53rd edn. (Cleveland: Chemical Rubber Co.), 155

Zanchet, A., Rodríguez-Lazcano, Y., Gálvez, Ó., et al. 2013, ApJ, 777, 26 